\title{
In vitro fermentation of oat bran obtained by debranning with a mixed culture of human faecal bacteria.
}

\author{
Gopal Kedia ${ }^{1}$, José A. Vázquez ${ }^{1,2}$ and Severino S. Pandiella ${ }^{1}$ * \\ ${ }^{1}$ School of Chemical Engineering and Analytical Science \\ The University of Manchester \\ Sackville Street, PO Box 88, Manchester M60 1QD, UK \\ ${ }^{2}$ Grupo de Reciclado y Valorización de Materiales Residuales \\ Instituto de Investigacións Mariñas (CSIC) \\ C/ Eduardo Cabello, 6. Vigo-36208. Galicia, Spain
}

Corresponding author E-mail: s.pandiella@manchester.ac.uk

Tel $+44(0) 1613064429$

$\mathrm{Fax}+44(0) 1613064399$

Keywords: Debranning, Faecal fermentation, Mixed culture fermentation, Oats, Prebiotic. 


\section{Abstract}

The prebiotic effect of oat samples was investigated by in vitro shake flask anaerobic fermentations with human faecal cultures. The oat bran fraction was obtained by debranning and was compared with other carbon sources such as whole oat flour, glucose and fructo oligosaccharide. The oat bran fraction showed a decrease in culturable anaerobes, clostridia and increase in bifidobacteria and lactobacilli populations. A similar pattern was observed in the fructo oligosaccharide. The butyrate production was higher in oat bran when compared to glucose and similar to fructo oligosaccharide. The production of propionate was higher in the two oat media than in fructo oligosaccharide and glucose, which could be used as energy source by the liver. This study suggests that the oat bran fraction obtained by debranning is useful for the gut ecosystem, and increases the population of beneficial bacteria of the indigenous gut microbiota. This medium also provides an energy source preferred by the colonocytes when it is metabolised by the gut flora.

\section{Introduction}

The intestinal microbiota of humans represent a complex ecosystem composed of 400-500 different species of metabolically active bacteria that impact strongly on the intestinal function and the health of host. Their number varies from $10^{11}$ to $10^{12}$ per gram of faeces. The anaerobic breakdown of substrates, such as undigested polysaccharides, resistant starch and fibre enhances the formation of lactic acid bacteria (LAB), but also of short-chain fatty acids (SCFA) as 
fermentation products. Increased production of short-chain fatty acids leads to a decrease in the $\mathrm{pH}$ of the colon and a low $\mathrm{pH}$ in the stool, which is associated with a reduced incidence of colon cancer in various populations [4].

Cereal grains contain essential nutrients like fibre, carbohydrates, proteins, vitamins, lipids and minerals. These components are found in specific parts of the grain and are not distributed uniformly. The cereal grains are made up of different layers, and in a simplified way they can be considered made of three parts: bran, endosperm and germ. The endosperm contains mostly starch and is the largest fraction comprising approximately $82 \%$ (dry basis) of a grain. The embryo is the portion of the grain that develops into the roots and shoot, and has the majority of the grain lipids, fats and sugars [21]. The bran fraction is responsible for protecting the kernel contents, and contains high levels of fibre, potassium, sodium, magnesium and calcium [13]. The bran layer is also a major source of proteins and which is mainly due to the aleurone layer. The aleurone layer is also an excellent source of amino acids for microbial fermentations. However, due to the thick indigestible cell walls of the bran layer the vitamins and proteins are not easily accessible when ingested by humans.

Originated from rice polishing, the technology of debranning (also referred to as pearling) has been integrated into some of the wheat milling industries to try to improve flour yield, to increase plant capacity and to simplify the mill flow $[2,3$, 22]. Debranning technology could also be used for the production of bran-rich 
fractions containing concentrated amounts of bran botanical constituents that could be diluted in other fractions produced from conventional milling [9]. This technology could be used to concentrate aleurone layer nutrients in a fraction rich in specific oligosaccharides that could be later used as a potential prebiotic source to increase the beneficial microbial population of the human gut.

Several epidemiological studies have shown differences in the intestinal microbiota between different population groups. The majority of the studies on the relationship of dietary fibre and intestinal flora have used animal models and in vitro models with pure cultures. However, in a mixed culture the strains will behave differently as compared to a pure culture [11, 12, 14]. In this paper a bran rich fraction of oats and whole oat flour have been studied for their prebiotic properties by using a mixed culture of human faecal bacteria. The results were compared with those obtained with a commercial fructooligosaccharide (FOS) with a studied prebiotic effect.

\section{Materials and Methods}

\section{Preparation of the oat fractions}

The whole oat flour was obtained by milling the grains in a hammer mill (Falling Number $A B$, England) fitted with a sieve of $850 \mu \mathrm{m}$ aperture size. The bran rich fraction was obtained by debranning using the Satake Abrasive Test Mill Model TM05C [22]. The pearling fractions obtained between 5 and $20 \mathrm{~s}$ of debranning 
was used in this study as the oat bran fraction and represents between 1-3\% of debranning of the whole kernel $[12,22]$.

\section{Batch culture fermentation}

Fermentation with human faecal inocula was carried out for 24 hours with different carbon sources to investigate the ability of the gut microbiota to utilize the oat bran fraction and whole oat flour. Controls included glucose, a well fermented substrate which supports the growth of most bacteria, and a fructo oligosaccharide obtained from chicory roots (FOS, Sigma) as a positive control of the prebiotic effect. The fermentation was performed in anaerobic conditions maintained by sparging the flask with oxygen-free nitrogen gas.

\section{Media}

The culture medium preparation $(50 \mathrm{~mL})$ was adopted from Vernazza et al. [20]. The media prepared contained $2 \mathrm{~g} / \mathrm{L}$ peptone water (Oxoid), $2 \mathrm{~g} / \mathrm{L}$ yeast extract (Oxoid), $0.1 \mathrm{~g} / \mathrm{L} \mathrm{NaCl}, 0.04 \mathrm{~g} / \mathrm{L} \mathrm{K}_{2} \mathrm{HPO}_{4}$ (Sigma), $0.04 \mathrm{~g} / \mathrm{L} \mathrm{KH}_{2} \mathrm{PO}_{4}$ (sigma), 0.01 g/L MgSO ${ }_{4} .7 \mathrm{H}_{2} \mathrm{O}$ (Sigma), $0.01 \mathrm{~g} / \mathrm{L} \mathrm{CaCl}_{2} .2 \mathrm{H}_{2} \mathrm{O}$ (Sigma), $2 \mathrm{~g} / \mathrm{L} \mathrm{NaHCO}_{3}$ (Sigma), 2 mL/L Tween 80 (sigma), 5 mg/L Hemin (Sigma), 10 mL/L Vitamin K1 (Sigma), $0.5 \mathrm{~g} / \mathrm{L}$ Cysteine- $\mathrm{HCl}$ (Sigma), $0.5 \mathrm{~g} / \mathrm{L}$ Bile Salts (Oxoid), $1 \mathrm{mg} / \mathrm{L}$ Resazurin (Sigma) and 5\% (w/v) of oat sample or control.

\section{Subjects}

Two healthy male volunteers aged 28 , provided the faecal samples. They had no history of gastrointestinal disorder, had avoided pro or prebiotic intake for at least 
one month prior to this study and had not taken antibiotics for 3 months before the experiment.

\section{Inocolum}

The faecal samples were collected in sealed plastic bags without excess air. Faeces were diluted 1:10 in phosphate buffered saline $(8 \mathrm{~g} / \mathrm{L} \mathrm{NaCl}, 0.2 \mathrm{~g} / \mathrm{L}$ of $\mathrm{KCl}, 1.15 \mathrm{~g} / \mathrm{L}$ of $\mathrm{Na}_{2} \mathrm{HPO}_{4}, 0.2 \mathrm{~g} / \mathrm{L}$ of $\mathrm{KH}_{2} \mathrm{PO}_{4}$ and $\mathrm{pH}$ was adjusted to 7.3). A 5\% $(v / v)$ of this inocolum was used for the in vitro trials in shake flask under anaerobic conditions.

\section{Determination of bacterial population in batch culture}

To determine the bacterial population, different selective media were used. MRS agar was used for Lactobacilli, BIM 25 for Bifidobacteria, SPS agar for Clostridia, Wilkins Chalgren Agar for culturable Anaerobes and MacConkey for Enterobacteriae. MRS agar plates for lactobacilli were cultured aerobically as lactobacilli are oxygen tolerant and hence other anaerobic bacteria will not grow in these conditions. The rest of the media were cultured anaerobically for 24-48 hours at $37^{\circ} \mathrm{C}$.

Direct quantification of total bacteria was carried out by the epifluorescent direct count method [10] using 4,6-diamidino-2-phenylindole (DAPI) staining. Samples were diluted 10 times with sterile PBS, and $0.5 \mathrm{~mL}$ of this suspension was fixed with $4.5 \mathrm{~mL}$ of $2 \%$ formaldehyde. They then were stained with DAPI (10 min, 
$1 \mu \mathrm{g} / \mathrm{mL})$ and filtered through polycarbonate membrane filters $(0.22 \mu \mathrm{m}$, Whatman International, Kent, UK). Bacteria were enumerated using an ocular graticule and 10 random fields per sample were counted.

\section{Short chain fatty acid (SCFA) analysis}

$2 \mathrm{~mL}$ of media were centrifuged at $4500 \mathrm{rpm}$ for 20 minutes to remove bacterial and particulate matter. The supernatant $(30 \mu \mathrm{L})$ was then subjected to HPLC using an Aminex HPX $-87 \mathrm{H}$ coloumn. The eluent was $0.005 \mathrm{~mol} / \mathrm{L}$ degassed $\mathrm{H}_{2} \mathrm{SO}_{4}$ at a flow rate of $0.6 \mathrm{~mL} / \mathrm{h}$ at $35^{\circ} \mathrm{C}$. Acetic acid, lactic acid, butyric acid and propionic acids were detected by UV absorbance at $210 \mathrm{~nm}$.

\section{Chemical analysis}

Ash and moisture contents were determined following the AACC Method 08-01 and 44-16, respectively [1]. Protein in the oat fraction was determined by multiplying the total Kjeldahl nitrogen by a factor of 6.25 . Total dietary fibre, soluble fibre and insoluble fibre were determined according to method of Prosky et al. [18]. $\beta$-glucan was determined according to the method of McCleary and Codd [15] using an assay kit K-BGLU from Megazyme (Megazyme International Ireland Ltd., Bray, County Wicklow, Ireland). Table 1 summarises the results from the chemical analyses of the bran rich oat fraction. Samples were taken by triplicate and the results reported are averages and standard deviations.

\section{Results}


Table 2 shows the growth of different bacteria groups in the four media tested. In all cases, the coefficients of variation of bacteria counts at initial time were lower than $5 \%$. Different population were able to grow to different degrees depending on the carbon source. The population of anaerobes decreases in the oat bran fraction and FOS, whereas they increase in glucose and whole oat flour media. The increment in Bifidobacteria was comparatively higher in FOS and bran rich oat media than in whole oat flour. The population of these bacteria decreased by $1 \log _{10} \mathrm{CFU} / \mathrm{mL}$ in glucose medium, and a similar pattern was observed for lactobacillus.

To obtain a general quantitative measure of the prebiotic effect, the prebiotic index $(P I)$ of the four media was calculated [17]. The $P I$ is defined as

$$
P I=\frac{\text { Bif }+ \text { Lac }- \text { Ana }- \text { Clos }}{\text { Total }}
$$

Where Bif, Ana, Lac, Clos and Total represent the ratio of cells of bifidobacterial, anaerobes, lactobacilli, clostridia and total bacteria obtained at a given time divided by the initial cell population (in $\log _{10} \mathrm{CFU} / \mathrm{mL}$ ). The equation assumes that an increase in the populations of bifidobacteria and/or lactobacilli has a positive effect while increments in anaerobes and clostridia are negative. Figure 1 shows the prebiotic index of the oat samples and controls as well as the corresponding confidence intervals $(\alpha=0.05 ; \mathrm{n}=2)[16]$. 
SCFA was analysed at the beginning and at end of the faecal fermentations and the results are shown in table 3. Lactate levels remains low in all the media after 24 hour of fermentation. No lactate was produced in glucose medium whereas both the oat bran and the flour produced levels of lactate comparable to those observed in FOS. Acetate was the main short chain fatty acid produced by human faecal bacteria after 24 hours. The glucose medium produced the highest maximum amounts of acetate followed by FOS, oat bran and whole oat flour. The butyrate level was lower in oat based broths as compared to FOS, whereas oat based media generated higher levels of propionate.

\section{Discussion}

To evaluate the prebiotic activity of new formulates it is necessary to analyse the evolution of mixed faecal populations of human origin. The behaviour of different bacteria during fermentation could vary depending on whether they are pure or mixed cultures due to synergistic, antagonistic and/or competitive effects. Fermentation in the gut is a complex process in which many metabolic pathways are carried out by different groups of bacteria [5]. The end product from one

group could be metabolised by others that cannot directly metabolise the original source substrate [8]. 
In the mixed culture with human faecal bacteria the oat bran fraction showed a prebiotic potential comparable to that found in commercially established prebiotic FOS. The population of bifidobacteria and lactobacilli in the oat bran significantly increased by 2.90 and $1.49 \log _{10} \mathrm{CFU} / \mathrm{mL}$, respectively. Similarly, in whole oat flour the population of bifidobacteria and lactobacillus increased by 2.07 and 0.82 $\log _{10} \mathrm{CFU} / \mathrm{mL}$, and in both cases the population of clostridia and anaerobes decreased. The $P I$ of the oat bran fraction was much higher than glucose after 24 hours and comparatively similar to the PI of FOS.

SCFA are the main metabolites formed in the intestine due to fermentation. They are an important source of energy for the human gut and can be transported to other tissues and organs of the human body [6]. Acetate is primarily used in muscle tissue whereas propionate is mainly used by the liver. Butyrate is the most important energy source for coloncytes, which should be constantly supplied to maintain good health $[7,19]$. In this study the butyrate production in the oat bran fraction $(6.98 \mathrm{mM})$ is higher than in the glucose medium $(5.71 \mathrm{mM})$ and almost similar to FOS $(7.04 \mathrm{mM})$. The production of propionate is higher in the oat bran fraction than in FOS and glucose media, which can be used as energy source by the liver. Lactate levels were very low in all the substrate tested. Lactate has a short life in the gut as it is a preferred electron sink product in anaerobic metabolism and could be quickly used by bacteria such as sulphate reducing bacteria. Our results suggest that the oat samples used in these 
experiments are beneficial for the gut ecosystem and when metabolised by the gut flora they can provide an energy source preferred by colonocytes.

\section{Conclusion}

This study investigated the effects of two different oat samples in the gut flora and showed that lactobacilli and bifidobacteria were able to grow in a faecal mixed culture. Enterobacteria, also considered beneficial for the gut, were also able to grow in these fractions. The population of negative bacteria such as clostridia and anaerobes considerably decreased in the oat bran and the SCFA production was comparable to that found in FOS, which is a well established prebiotic. The Prebiotic index suggests that an oat bran rich fraction obtained by debranning could be used as a prebiotic ingredient and could have a functional effect comparable to some of the commercial prebiotics. However, human intervention studies should be used to confirm the efficacy or equivalence of the oat bran fractions to existing prebiotics.

\section{References}

1. AACC (1995) Methods from American Association of Cereal Chemists: Approved Methods of the Association, Ninth Edition, The Association: St. Paul, MN, USA

2. Bradshaw J (2004) Debranning. Grain and Feed Mill Tech (July-August):10-13

3. Bradshaw J (2005) Developments in semolina milling. Grain and Feed Mill Tech (July-August):14-17

4. Caplice E, Fitzgerald GF (1999) Food fermentations: Role of microorganisms 
in food production and preservation. Int J Food Microb 50:131-149

5. Cummings J (1984) Colonic absorption: the importance of short chain fatty acids in man. Scand J Gastroent 93:89-99

6. Cummings JH, Macfarlane GT (1991) Production and metabolism of shortchain fatty acids in humans. Tenth Ross Confer on Medical Res

7. Englyst H, Hay S, Macfarlane G (1987) Polysaccharide breakdown by mixed populations of human faecal bacteria. FEMS Microb Ecol 95:163-169

8. Gibson G, Roberfroid M (1995) Dietary modulation of the human colonic microbiota: Introducing the concept of prebiotics. J Nutri 125:1401-1412

9. Handelman GJ, Cao G, Walter MF, Nightingale ZD, Paul GL, Prior RL (1999) Antioxidant capacity of oat (Avena sativa L.) extracts. Inhibition of low-density lipoprotein oxidation and oxygen radical absorbance capacity. J Agric Food Chem 47:4888-4893

10. Hobbie JE, Daley RJ, Jasper S (1977) Use of nucleopore filters for counting bacteria by fluorescence microscopy. Appl Environ Microbiol 33:1225-1228.

11. Kedia G, Wang R, Patel H, Pandiella SS (2007) Use of mixed cultures for the fermentation of cereal-based substrates with potential probiotic properties. Proc Biochem 42:65-70

12. Kedia G, Vázquez JA, Pandiella SS (2008) Evaluation of the fermentability of oat fractions obtained by debranning using lactic acid bacteria. J Appl Microbiol DOI: $10.1111 / j .1365-2672.2008 .03864 . x$

13. MacMasters MM, Hinton JJC, Bradbury D (1971) Microscopic structure and composition of the wheat kernel. Wheat: Chemistry and Technology. Y. Pomeranz. Minnesota, AACC

14. Mandalari G, Nueno Palop C, Tuohy K, Gibson GR, Bennett RN, Waldron $\mathrm{KW}$, Bisignano G, Narbad A, Faulds CB (2007) In vitro evaluation of the prebiotic activity of a pectic oligosaccharide-rich extract enzymatically derived from bergamot peel. Appl Microb Biotech 73:1173-1179

15. McCleary BV, Codd R (1991) Measurement of (1-3) (1-4) beta glucan in barley and oats: A streamlined enzymic procedure. J Sci Food Agric 55:303-312

16. Motulsky H (1995) Intuitive Biostatistics. Motulsky, H, Ed., Oxford University Press, New York, pp 285-286

17. Palframan R, Gibson G, Rastall R (2003) Development of a quantitative tool 
for the comparison of the prebiotic effect of dietary oligosaccharides. Lett Appl Microb 37:281-284

18. Prosky L, Asp NG, Schweizer T, DeVries J, Furda I (1988) Determination of insoluble, soluble, and total dietary fibre in foods and food products: interlaboratory study. J Assoc Offic Anal Chem 71:1017-1023

19. Titgemeyer E, Bourquin L, Fahey G, Garleb K (1991) Fermentability of various fibre sources by human fecal bacteria in vitro. Am J Clin Nutri 53:14181424

20. Vernazza CL, Gibson GR and Rastall R (2005) In vitro fermentation of chitosan derivatives by mixed cultures of human faecal bacteria. Carbohyd Polym 60:539-545

21. Wang R (1999) Continuous production of a generic fermentation feedstock from whole wheat flour. Department of Chemical Engineering. Manchester, UMIST

22. Wang R, Koutinas A, Campbell G (2007) Effect of pearling on dry processing of oats. J Food Eng 82:369-376 


\section{Tables and figure legends}

Table 1 Chemical analysis of the oat bran rich fraction. Results are expressed as average \pm standard deviation $(n=3)$.

Table 2 Changes in cell population $\left(\log _{10} \mathrm{CFU} / \mathrm{mL}\right)$ in the batch faecal fermentations. Results are expressed as average \pm standard deviation $(n=2)$.

Table 3 Short chain fatty acid levels ( $\mathrm{mM})$ in the batch faecal fermentations.

Figure 1 Prebiotic Index and confidence intervals $(\alpha=0.05 ; \mathrm{n}=2)$ of the oat samples and controls. 


\section{Table 1}

\begin{tabular}{ll}
\hline Moisture $(\%)$ & $6.24 \pm 0.13$ \\
Ash $(\%, \mathrm{db})$ & $4.76 \pm 0.35$ \\
Starch $(\%, \mathrm{db})$ & $7.72 \pm 0.21$ \\
$\beta$-Glucan $(\%, \mathrm{db})$ & $9.43 \pm 0.32$ \\
Total dietary fibre $(\%, \mathrm{db})$ & $32.34 \pm 1.21$ \\
Soluble Fiber $(\%, \mathrm{db})$ & $14.56 \pm 0.76$ \\
Insoluble fiber $(\%, \mathrm{db})$ & $17.46 \pm 0.84$ \\
Protein $(\%, \mathrm{db})$ & $23.09 \pm 2.12$ \\
\hline
\end{tabular}




\section{Table 2}

\begin{tabular}{|c|c|c|c|c|c|}
\hline & \multirow{2}{*}{$\begin{array}{c}\text { Time } \\
\text { (hr) }\end{array}$} & \multicolumn{4}{|c|}{ Total Cell count $\log _{10}$ CFU/mL } \\
\hline & & Glucose & Oat Bran Fraction & Whole Oat Flour & FOS \\
\hline \multirow{4}{*}{ Total Bacteria } & 0 & $9.72 \pm 0.12$ & $9.68 \pm 0.17$ & $9.71 \pm 0.22$ & $9.74 \pm 0.15$ \\
\hline & 5 & $9.64 \pm 0.18$ & $9.52 \pm 0.14$ & $9.55 \pm 0.08$ & $9.42 \pm 0.16$ \\
\hline & 10 & $10.45 \pm 0.11$ & $9.21 \pm 0.09$ & $9.40 \pm 0.15$ & $9.31 \pm 0.08$ \\
\hline & 24 & $11.24 \pm 0.16$ & $8.98 \pm 0.13$ & $9.24 \pm 0.11$ & $9.12 \pm 0.14$ \\
\hline \multirow{4}{*}{ Bifidobacteria } & 0 & $7.82 \pm 0.07$ & $7.92 \pm 0.18$ & $7.77 \pm 0.14$ & $7.88 \pm 0.07$ \\
\hline & 5 & $7.58 \pm 0.12$ & $8.04 \pm 0.06$ & $7.69 \pm 0.07$ & $7.91 \pm 0.10$ \\
\hline & 10 & $7.32 \pm 0.18$ & $9.44 \pm 0.14$ & $8.82 \pm 0.18$ & $9.22 \pm 0.22$ \\
\hline & 24 & $6.85 \pm 0.14$ & $10.82 \pm 0.22$ & $9.84 \pm 0.11$ & $10.93 \pm 0.17$ \\
\hline \multirow{4}{*}{ Lactobacillus } & 0 & $7.71 \pm 0.08$ & $7.47 \pm 0.13$ & $7.52 \pm 0.08$ & $7.64 \pm 0.10$ \\
\hline & 5 & $7.41 \pm 0.11$ & $7.54 \pm 0.17$ & $7.24 \pm 0.08$ & $7.61 \pm 0.13$ \\
\hline & 10 & $7.39 \pm 0.09$ & $8.24 \pm 0.05$ & $7.96 \pm 0.07$ & $8.45 \pm 0.06$ \\
\hline & 24 & $6.64 \pm 0.05$ & $8.96 \pm 0.17$ & $8.34 \pm 0.11$ & $9.24 \pm 0.07$ \\
\hline \multirow{4}{*}{ Clostridia } & 0 & $6.85 \pm 0.12$ & $6.72 \pm 0.08$ & $6.62 \pm 0.11$ & $6.92 \pm 0.13$ \\
\hline & 5 & $6.61 \pm 0.17$ & $6.51 \pm 0.05$ & $6.59 \pm 0.09$ & $6.41 \pm 0.08$ \\
\hline & 10 & $7.91 \pm 0.13$ & $6.21 \pm 0.13$ & $6.67 \pm 0.08$ & $6.14 \pm 0.11$ \\
\hline & 24 & $9.54 \pm 0.12$ & $5.83 \pm 0.08$ & $7.04 \pm 0.11$ & $5.54 \pm 0.09$ \\
\hline \multirow{4}{*}{ Anaerobes } & 0 & $8.48 \pm 0.07$ & $8.27 \pm 0.14$ & $8.56 \pm 0.13$ & $8.41 \pm 0.06$ \\
\hline & 5 & $8.50 \pm 0.10$ & $8.51 \pm 0.07$ & $8.46 \pm 0.11$ & $8.37 \pm 0.08$ \\
\hline & 10 & $9.52 \pm 0.14$ & $8.05 \pm 0.18$ & $8.31 \pm 0.10$ & $7.82 \pm 0.15$ \\
\hline & 24 & $10.87 \pm 0.08$ & $7.42 \pm 0.06$ & $8.67 \pm 0.15$ & $7.01 \pm 0.12$ \\
\hline \multirow{4}{*}{ Enterobacteria } & 0 & $8.34 \pm 0.07$ & $8.24 \pm 0.17$ & $8.13 \pm 0.11$ & $8.03 \pm 0.15$ \\
\hline & 5 & $8.01 \pm 0.08$ & $8.57 \pm 0.12$ & $8.04 \pm 0.14$ & $8.21 \pm 0.20$ \\
\hline & 10 & $7.85 \pm 0.13$ & $8.76 \pm 0.18$ & $8.18 \pm 0.09$ & $8.74 \pm 0.11$ \\
\hline & 24 & $6.32 \pm 0.11$ & $9.12 \pm 0.08$ & $8.34 \pm 0.15$ & $8.94 \pm 0.17$ \\
\hline
\end{tabular}




\section{Table 3}

\begin{tabular}{|l|c|c|c|c|c|c|c|c|}
\hline \multirow{2}{*}{ SCFA } & \multicolumn{2}{|c|}{ Glucose } & \multicolumn{2}{c|}{ FOS } & \multicolumn{2}{c|}{ Oat Bran Fraction } & \multicolumn{2}{c|}{ Whole Oat Flour } \\
\cline { 2 - 9 } & $\mathrm{t}_{0}$ & $\mathrm{t}_{24}$ & $\mathrm{t}_{0}$ & $\mathrm{t}_{24}$ & $\mathrm{t}_{0}$ & $\mathrm{t}_{24}$ & $\mathrm{t}_{0}$ & $\mathrm{t}_{24}$ \\
\hline Lactate & 0.00 & 0.00 & 0.00 & 0.10 & 0.00 & 0.41 & 0.00 & 0.56 \\
\hline Acetate & 0.08 & 36.09 & 0.12 & 32.14 & 0.10 & 30.11 & 0.14 & 28.14 \\
\hline Propionate & 0.20 & 14.11 & 0.41 & 13.67 & 0.21 & 15.68 & 0.18 & 14.71 \\
\hline Butyrate & 0.08 & 5.71 & 0.09 & 7.04 & 0.09 & 6.98 & 0.08 & 5.94 \\
\hline
\end{tabular}


Figure 1

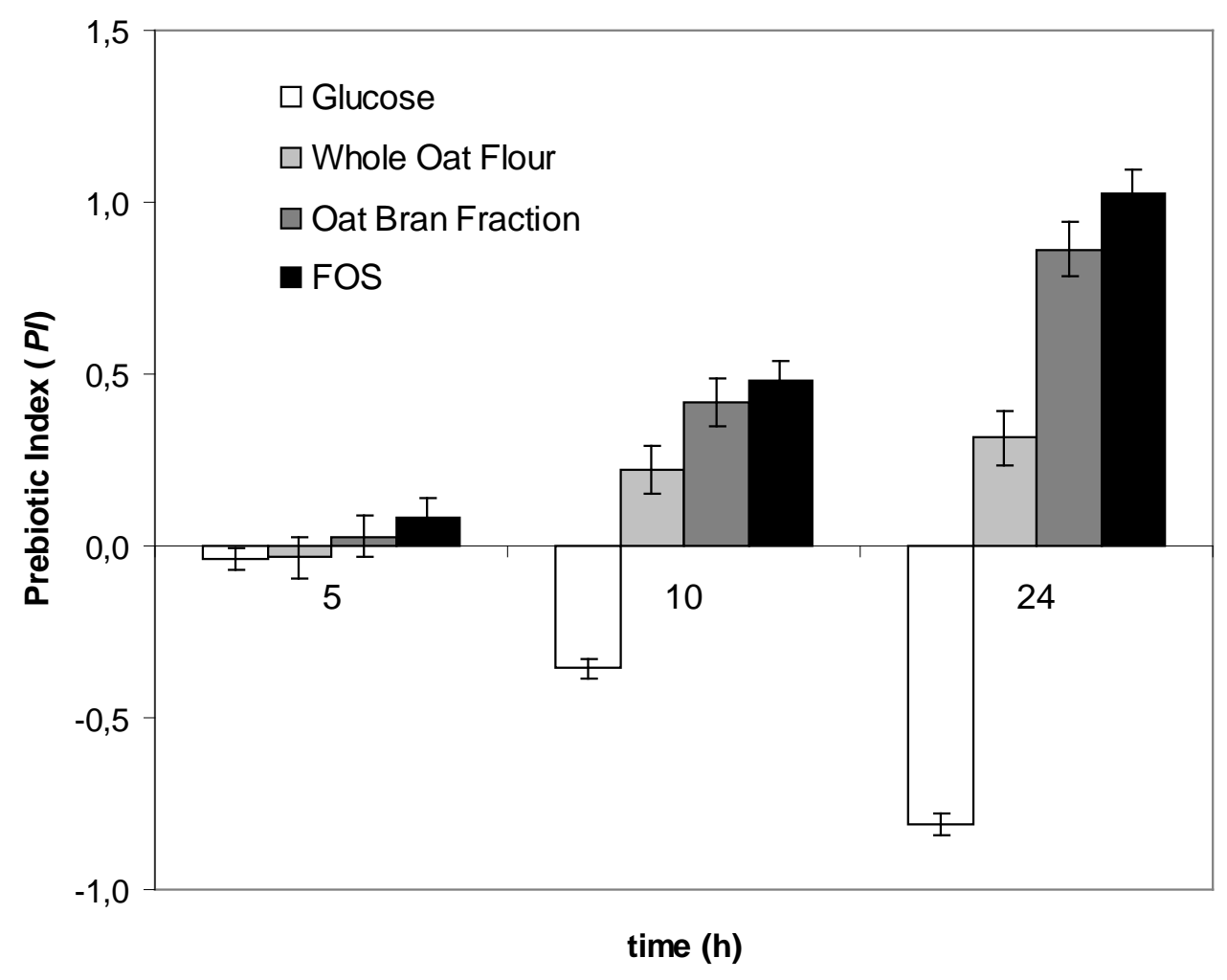

